Revue internationale P.M.E.

Économie et gestion de la petite et moyenne entreprise

\title{
Les pratiques commerciales des petites entreprises du tourisme sportif de nature : entre rationalité marketing et logiques sociales des dirigeants
}

\section{Malek Bouhaouala et Pascal Chantelat}

Volume 15, numéro 1, 2002

URI : https://id.erudit.org/iderudit/1008799ar

DOI : https://doi.org/10.7202/1008799ar

Aller au sommaire du numéro

Éditeur(s)

Presses de l’Université du Québec

ISSN

0776-5436 (imprimé)

1918-9699 (numérique)

Découvrir la revue

Citer cet article

Bouhaouala, M. \& Chantelat, P. (2002). Les pratiques commerciales des petites entreprises du tourisme sportif de nature : entre rationalité marketing et logiques sociales des dirigeants. Revue internationale P.M.E., 15(1), 11-38. https://doi.org/10.7202/1008799ar
Résumé de l'article

Cet article analyse l'activité commerciale des PE-TPE du tourisme sportif. Il vise à dépasser l'explication néoclassique des comportements commerciaux en tenant compte des variables sociologiques inhérentes aux dirigeants. L'hypothèse défendue ici suppose que les pratiques commerciales sont relatives aux logiques d'action des dirigeants, à leur manière de concevoir le marché, le client, le sport et la nature, ainsi qu'à leurs projets socioprofessionnels. En effet, la considération de ces éléments permet de comprendre les orientations stratégiques des PE-TPE et d'expliquer des comportements commerciaux pouvant parfois paraître comme irrationnels ou inefficaces. Cette étude est fondée sur une enquête qualitative par entretiens semi-directifs réalisés auprès de 28 dirigeants de PE-TPE situées dans le massif du Vercors (Alpes françaises). Les résultats mettent en évidence quatre types d'offre de services se distinguant par des pratiques commerciales et des logiques d'action spécifiques. Il s'agit de l'offre : a) artisanale passionnée, b) entrepreneuriale, c) sociomanagériale et d) traditionaliste. 


\title{
Les pratiques commerciales des petites entreprises du tourisme sportif de nature: entre rationalité marketing et logiques sociales des dirigeants
}

Malek BOUHAOUALA

Université de Lille II

Pascal CHANTELAT

Université Claude-Bernard Lyon I

\section{MOTS CLÉS}

\section{PE-TPE - Dirigeants - Actions commerciales - Logiques d'action Tourisme sportif de nature}

\begin{abstract}
RÉSUMÉ
Cet article analyse l'activité commerciale des PE-TPE du tourisme sportif. Il vise à dépasser l'explication néoclassique des comportements commerciaux en tenant compte des variables sociologiques inhérentes aux dirigeants. L'hypothèse défendue ici suppose que les pratiques commerciales sont relatives aux logiques d'action des
\end{abstract}

\section{LES AUTEURS}

MaleK Bouhaouala est maître de conférences à l'Université du Droit et de la Santé de Lille II, chercheur au Sport, identités et culture (SIC) de Lille II et Associé au laboratoire Études et recherches sur l'offre sportive (EROS) de Grenoble I. II est aussi membre du Réseau des chercheurs et experts en sports de montagne et de nature et membre de l'Académie de l'entrepreneuriat. II a publié des articles concernant les PE-TPE, les entrepreneurs et la régulation des marchés locaux dans le domaine du tourisme et des services sportifs. Adresse: Université du Droit et de la Santé de Lille II, Faculté des sciences du sport, 9, rue de l'Université, Ronchin 59790, France. Téléphone :33615104757 Courriel :<mbouhaouala@mailsc.univ-lille2.fr>.

Pascal Chantelat est maître de conférences à l'Université Claude-Bernard Lyon I et est également chercheur au Centre de recherches sur l'innovation sportive (CRIS) de Lyon I. II a publié des articles sur les industries d'articles de sport, l'économie des clubs sportifs et la professionnalisation dans le domaine du sport. Par ailleurs, il collabore à des travaux dans le domaine du tourisme et des loisirs sportifs. 
dirigeants, à leur manière de concevoir le marché, le client, le sport et la nature, ainsi qu'à leurs projets socioprofessionnels. En effet, la considération de ces éléments permet de comprendre les orientations stratégiques des PE-TPE et d'expliquer des comportements commerciaux pouvant parfois paraître comme irrationnels ou inefficaces. Cette étude est fondée sur une enquête qualitative par entretiens semi-directifs réalisés auprès de 28 dirigeants de PE-TPE situées dans le massif du Vercors (Alpes françaises). Les résultats mettent en évidence quatre types d'offre de services se distinguant par des pratiques commerciales et des logiques d'action spécifiques. II s'agit de l'offre: a) artisanale passionnée, b) entrepreneuriale, c) sociomanagériale et d) traditionaliste.

\section{ABSTRACT}

This paper deals with small and very small firms (SF-VSF) business activity in outdoor tourism services. The objective is to analyze these latter as stemming from managers logic of action and not as marketing rationalization of behaviors thinks they should be. Therefore sociological approach is used in order to point out the role of managers' subjectivity and goals in the orientation of their firms business strategies. This research defends the hypothesis of the variety of SF-VSF business practices. Based on semi-structured interviews of 28 small firms' managers in the massif of Vercors (French Alps). This research points out typology of SF-VSF trading action corresponding to managers' practical logics of action. This typology comprises four types of marketing recognized by: 1) passionate craftsman, 2) entrepreneurial, 3) socio-managerial and 4) traditionalist supplies.

\section{RESUMEN}

Este artículo se ocupa de pequeñas y muy pequeñas firmas la actividad económico en servicios de turismo exteriores. El objetivo es de analizar este éste como la peladura de gerentes la lógica de acción y no como la racionalización de control de comercialización de comportamientos piensa que ellos deberían ser. El acercamiento Por lo tanto sociológico es usado para indicar el papel de la subjetividad de los gerentes y objetivos en la orientación de sus estrategias de negocio de firmas. Esta investigación defende las hipótesis de la variedad de pequeñas firmas prácticas de negocio. Basado en las entrevistas semi-estructuradas de los gerentes de 28 pequeñas firmas en el macizo de Vercors (Alpes franceses). Esta investigación indica la tipología de pequeñas firmas la acción comercial que corresponde a las lógicas prácticas de los gerentes de acción. Esta tipología comprende cuatro tipos de control de comercialización aprobado por: 1) artesano apasionado, 2) emprendedor, 3) socio-directivo y 4) provisiones de tradicionalista.

\section{ZUSAMMENFASSUNG}

Dieser Artikel analysiert die Handelsaktivitäten der Kleinbetriebe aus dem Bereich Sport/Tourismus. Er zielt ab die neoklassische Erklärung des Handelsverhaltens unter Berücksichtigung verschiedener soziologischen Variablen der Unternehmensleiter zu erfassen. Die vertretene Hypothese vermutet, dass die Handelspraktiken in Beziehung stehen zu den Handlungen der Unternehmensleiter, wie sie den Markt, den Kunden, den Sport und die Natur und ihre sozial-unternehmerischen

Revue internationale P.M.E., vol. 15, $\mathrm{n}^{\circ} 1,2002$ 
Projekte wahrnehmen. Die Berücksichtigung von diesen Elementen erlaubt die strategische Orientierung der Kleinbetriebe zu verstehen und erklärt das oft als irrationell und uneffizient beurteilte Verhalten. Diese Studie ist aufgebaut auf einer qualitativen Untersuchung bei 28 Unternehmensleitern von Kleinbetrieben im Massiv von Vercors (Französische Alpen). Die Ergebnisse heben vier unterschiedliche Dienstleistungstypen hervor, die sich in den Handelspraktiken und spezifischer Handlungslogik unterscheiden: Es handelt sich um den passionierten Gewerbler, den Unternehmer, den sozialen Manager und den Traditionalisten.

\section{Introduction}

L'idée de base de cet article est que la petite entreprise constitue l'affaire du dirigeant et que sa finalisation (orientation stratégique, organisation, mode de production, etc.) et son activité commerciale sont liées à la logique d'action de ce dernier (Julien et Marchesnay, 1996). Il est donc permis de considérer que le profit et la satisfaction du client peuvent être considérés par le dirigeant comme des moyens pour atteindre des objectifs non économiques (Bouhaouala, 2001 ; Boutillier et Uzunidis, 1995). Partant, on peut supposer que les pratiques commerciales de ce dernier ne répondent pas exclusivement aux principes du marketing management ${ }^{1}$ qui constitue une vision marchande de l'activité en entreprise (Marion, 1995). Il convient donc de placer les dirigeants, leurs logiques d'action et leurs pratiques professionnelles au centre de l'analyse de l'action commerciale des petites et très petites entreprises (PE-TPE). L'analyse peut alors se faire du point de vue de la sociologie dont l'apport peut être non négligeable à ce niveau (Marion, 1995). En effet, cette auteure considère tout phénomène économique comme fondamentalement social (Freund, 1966; Weber, 1905, 1922). De ce fait, les activités commerciales ne seront pas analysées ici exclusivement du point de vue de la rationalisation microéconomique ou marketing, mais à partir des pratiques et logiques des dirigeants.

L'hypothèse selon laquelle le dirigeant agit prioritairement en fonction de la recherche systématique du profit, de la satisfaction du «client roi » et que l'entreprise a pour objectif de répondre exclusivement aux besoins de la demande constituent «des généralisations exacerbées » et des caractéristiques qui ne sont pas applicables universellement (Lovelock et Lapert, 1999). Dès lors, cette hypothèse devient une position difficile à maintenir (Marion, 1999) en particulier pour les PE-TPE du tourisme sportif ${ }^{2}$ (Bouhaouala, 2001).

1. «Le marketing management est un ensemble de décisions (relatives au produit, au prix, à la distribution et à la publicité) et d'instruments permettant la mise en œuvre d'un plan marketing » ( $c f$. Marion, 1995). Le marketing mix en est donc un des éléments clés.

2. Le tourisme sportif de nature combine un ensemble de services périphériques (hébergement, restauration, animation, séminaires, transport) autour d'un service principal

Revue internationale P.M.E., vol. 15, n ${ }^{\circ} 1,2002$ 
Fort de ce constat, cet article vise à mettre en évidence le rôle des dirigeants ainsi que la place qu'ils accordent à leur propre projet socioprofessionnel, objectifs et conceptions de la nature, du sport, du client, du marché... dans la détermination de leur activité commerciale. Il s'agit donc de développer une approche multidisciplinaire expliquant l'influence des dimensions non économiques sur l'action commerciale des PE-TPE du tourisme sportif dans laquelle le marketing mix $^{3}$ constitue une première grille de lecture (Eiglier et Langeard, 1991).

\section{La diversité des objectifs des dirigeants de la petite entreprise}

Les buts des dirigeants peuvent porter davantage sur le choix d'un métier passionnant, la recherche d'une qualité de vie ou de la convivialité (Berett, Burton et Slack, 1993 ; Bouhaouala et Chifflet, 2001 ; Collins et Randolph, 1991 ; Lahlou, Maffre et Moati, 1991; Callot, 1999) que sur l'unique maximisation des profits. La recherche de l'indépendance peut également conduire à un refus de croissance de l'entreprise (taille, part de marché, etc.) perçue comme une menace pour l'atteinte des buts initiaux des dirigeants-propriétaires (Bouhaouala, 2001; Boutillier et Uzunidis, 1995 ; Gresle 1981 ; Marchesnay, 1998 ; Callot, 1999).

Compte tenu de la multiplicité des objectifs (Marchesnay, 1997, 1998) et des orientations stratégiques (Bertrand, 1996) des dirigeants, leurs comportements ne sont pas «naturellement» orientés par une rationalité formelle (c'est-à-dire managériale). On peut même supposer que, globalement, les dirigeants de PE-TPE adoptent des attitudes éloignées, voire contraires à la logique du marché et à la rationalité économique (Gresle, 1981; Zarca, 1986; Callot, 1999).

Dans le domaine des sports de pleine nature et de montagne, l'étude de San José, Fidelle et Bruant (1995) a montré l'impact de la subjectivité des prestataires sportifs sur l'activité commerciale de leurs entreprises. En effet, pour les écoles de parapente, les modes de production et de commercialisation des services varient en fonction de la conception du risque d'accidents qu'ont les moniteurs prestataires.

constitué de un ou de plusieurs sports se déroulant en milieu naturel (montagne, eau vive, mer, etc.). De ce fait, le processus de servuction en tourisme sportif de nature nécessite la participation d'au moins deux parties (client et prestataire) et l'usage de produits (p. ex., équipements sportifs, structures d'accueil) dans un milieu naturel avec tout ce que ce dernier comporte comme conditions indispensables à ce type de services (paysage, évasion, dénivelé, neige, cour d'eau, falaise, etc. ; $c f$. Eiglier et Langeard, 1991 ; Lovelock et Lapert, 1999).

3. Le marketing mix renvoie à l'ensemble des éléments plus communément désignés par les 4P dont dispose expressément une organisation pour concevoir une offre de services en vue d'un positionnement commercial (Cliquet, 1999; Flipo et al., 1980).

Revue internationale P.M.E., vol. 15, nº 1, 2002 
On est donc en droit de penser que les conceptions et les objectifs des dirigeants orientent leurs choix économiques et démarches commerciales. Cela confirme l'importance de la prise en compte du rôle de l'acteur (et de sa subjectivité) dans la compréhension de l'action commerciale de la petite entreprise. Partant, il convient de reconstruire l'ensemble des significations (conceptions, objectifs et motifs) relatives aux activités professionnelles et économiques des dirigeants. Il s'agit ensuite de mettre en relation le marketing mix des entreprises avec la subjectivité des dirigeants afin de déduire et de comprendre les logiques d'action qui les sous-tendent.

\section{Une approche compréhensive}

Les recherches qui ont porté sur les artisans français (Gresle, 1981 ; Zarca, 1986) ou sur les entrepreneurs (Boutillier et Uzunidis, 1995, 1999) montrent que ces derniers, caractérisés par «l'idéologie de l'indépendance» (Gresle, 1981), «une mentalité artisanale traditionnelle » (Zarca, 1986), « un style de vie » ou « un système moral » (Boutillier et Uzunidis, 1995), adoptent des comportements économiques plus en rapport avec leur propre logique sociale qu'avec les paramètres du marché.

Ces travaux (Boutillier et Uzunidis, 1995, 1999; Gresle, 1981; Gislain et Steiner, 1995 ; Steiner, 1998; Weber, 1922 ; Zarca, 1986) constituent donc un point de départ pour construire un modèle explicatif applicable à l'analyse de comportements économiques des dirigeants de PE-TPE. Ce dernier sera fondé sur le concept désigné par le terme de «micromentalité ${ }^{4}$ » qui fait référence à une échelle sociale plus réduite que celle à laquelle renvoie la notion de mentalités ou d'ethos qui, en général, font référence à des groupes sociaux entiers (Lloyd, 1996; Mucchielli, 1985). Les micromentalités sont ainsi définies comme un concept synthétique introduisant, dans l'analyse des phénomènes économiques, un ensemble de conceptions et de valeurs, d'objectifs et de motifs d'action non obligatoirement cohérents avec le raisonnement abstrait de l'homo æeconomicus. Ces éléments contribuent à créer une vision subjective du monde et produisent des comportements compréhensibles à partir de logiques sociales intégrant des variables non économiques (Steiner, 1998). Cette dernière est une vision globale et pragmatique de l'activité économique; globale, dans le sens où l'activité économique n'est pas extraite de la vie sociale des acteurs, et pragmatique, car elle produit des logiques d'actions pratiques et non des réflexions abstraites (Steiner, 1998). En d'autres

4. La micromentalité des dirigeants est conceptualisée ici à partir de l'adaptation de la notion de mentalités (Gislain et Steiner, 1995) ou d'ethos (Weber, 1922) à un espace social restreint. En effet, l'idée que les mentalités ou l'éthique influencent les comportements de groupes sociaux importants n'est pas une nouveauté en soi (Gislain et Steiner, 1995 ; Steiner, 1998).

Revue internationale P.M.E., vol. 15, nº 1, 2002 
termes, les acteurs sociaux sont susceptibles d'agir selon des logiques contradictoires à l'égard d'une rationalité matérielle au plan utilitaire (Lahire, 1998; Steiner, 1998). Les micromentalités et les logiques d'action qu'elles produisent permettent donc d'expliquer les comportements à partir d'une détermination relative et significative.

Les micromentalités ${ }^{5}$ sont donc susceptibles d'orienter les actions commerciales des dirigeants qui privilégient leur projet socioprofessionnel aux paramètres du marché. Leurs actions commerciales peuvent ne pas être uniquement fondées sur un calcul économique des coûts et des avantages; elles peuvent être fondées sur des logiques particulières repérables dans leurs démarches de : 1 ) production (élaboration des produits et fixation des prix) et 2) commercialisation (publicité et vente; $c f$. figure 1$)$.

FIGURE 1

Modèle d'analyse de la logique d'action

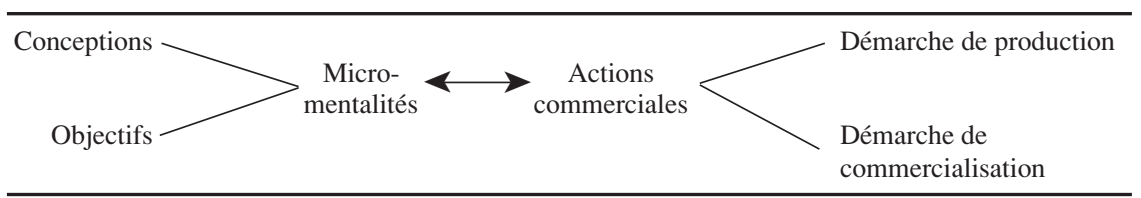

\section{Les micromentalités : un concept opératoire}

Pour reconstruire les logiques d'action des dirigeants, il est nécessaire d'identifier, d'une part, les micromentalités à partir d'abord de leurs conceptions de l'économie, du marché, du client, de l'activité professionnelle, de l'entreprise, du produit, du service sportif... et ensuite de leurs objectifs et motifs d'action qui renvoient aux projets social, financier et professionnel. D'autre part, il est nécessaire de repérer les pratiques commerciales des dirigeants à travers leurs démarches de «production » et de commercialisation par l'analyse des quatre éléments du marketing mix (Kotler et Dubois, 1989) de leur offre : le produit, le prix, la publicité et la vente (cf. tableau 1).

La mise en relation des pratiques commerciales avec les conceptions et les objectifs des dirigeants permet d'abord de comprendre et d'expliquer leur logique

5. Le concept de micromentalités ne renvoie pas à une pure subjectivité individuelle ou à la structure mentale des acteurs. Il permet de dépasser ces dernières, tout en suivant une approche compréhensive, et vise à reconstruire des ensembles de significations propres à des groupes d'acteurs (Bentabe, Michun et Trouvé, 1999). Il insiste donc sur le caractère sociologique et individualisant de leurs logiques d'action et de leur rapport au monde marchand et professionnel. 
d'action et, ensuite, de rendre compte d'éventuelles incohérences par rapport au modèle du mercaticien, donc d'une possible diversité des démarches commerciales des PE-TPE du tourisme sportif de nature.

TABLEAU 1

Dimension, thèmes et indicateurs d'analyse

\begin{tabular}{|c|c|c|}
\hline Thèmes & Dimensions & Indicateurs \\
\hline MicROMENTALITÉ & $\begin{array}{l}\text { 1) Conceptions } \\
\text { 2) Objectifs et motifs }\end{array}$ & $\begin{array}{l}\text { - Du travail, de l'argent, du sport, de la nature } \\
\text { (montagne, Vercors), du marché, du client... } \\
\text { - } \quad \text { Sociaux, financiers et professionnels. }\end{array}$ \\
\hline $\begin{array}{l}\text { PRATIQUES } \\
\text { COMMERCIALES }\end{array}$ & $\begin{array}{l}\text { 1) Démarche de conception- } \\
\text { production }\end{array}$ & $\begin{array}{l}\text { 1.1 Élaboration des produits (critères } \\
\text { commerciaux ou personnels, expérience, } \\
\text { plaisir, demande, etc.). } \\
\text { 1.2 Fixation des prix (coûts, marché, concurrence, } \\
\text { revenus, etc.). } \\
\text { 2.1 Communication et publicité (importance, } \\
\text { portée, coûts, arguments, supports, etc.). } \\
\text { 2.2 Commercialisation et vente (vente directe, par } \\
\text { intermédiaire, rapport au client, etc.). }\end{array}$ \\
\hline
\end{tabular}

\section{Recueil et traitement des données}

Cette étude présente une partie des résultats recueillis lors d'une recherche doctorale sur les petites entreprises et les entrepreneurs du tourisme sportif de nature. L'enquête s'est déroulée au cours de l'été 1998 (juin-août) auprès de 28 dirigeants de petites et très petites entreprises du tourisme sportif sur le massif du Vercors. Ce massif regroupant un ensemble d'entreprises assez diversifié du point de vue du statut juridique et des activités commercialisées liées au tourisme sportif de nature constitue de ce fait un terrain d'études privilégié. L'ensemble des 28 entreprises a été déterminé en tenant compte de la diversité des PE-TPE en termes d'activité principale (service sportif, accueil, etc.), de statut juridique et de taille (cf. figure 2).

En raison du rôle accordé à la subjectivité des acteurs dans l'orientation des pratiques économiques, l'approche qualitative et l'analyse thématique (Huberman et Mills, 1991) semblent ici adaptées. Des entretiens semi-directifs d'une heure ont été réalisés auprès de 28 dirigeants et organisés en deux grands thèmes nécessaires à :

a) la reconstruction des micromentalités des dirigeants. Les questions posées visaient à rendre compte à la fois de la dimension subjective (conceptions et objectifs) de leur comportement et de leur positionnement (opinions et jugements de valeur) à l'égard du monde marchand et professionnel ;

Revue internationale P.M.E., vol. 15, nº 1, 2002 
FiguRE 2

\section{Choix des entreprises enquêtées}

\begin{tabular}{|c|c|c|}
\hline $\begin{array}{l}\text { Services commercialisés } \\
\text { Services sportifs, d'accueil, } \\
\text { organisation }\end{array}$ & $\begin{array}{l}\text { Taille en effectif } \\
\text { Moins de } 50 \text { salarisés }\end{array}$ & $\begin{array}{c}\text { Statuts juridiques } \\
\text { EURL, SARL, SA, SEM, } \\
\text { Association Loi } 1901\end{array}$ \\
\hline 4 & $\downarrow$ & $\checkmark$ \\
\hline \multicolumn{3}{|c|}{$\begin{array}{l}\text { - } \mathbf{6} \text { entreprises des sports de plein air (EURL et SARL, multiactivités sportives et organisation), } \\
\text { - } \mathbf{2} \text { centres d'équitation (EURL, monoactivité sportive), } \\
\text { - } \mathbf{1} \text { agence de voyages (SARL, organisation), } \\
\text { - } \mathbf{4} \text { travailleurs indépendants (EURL, multiactivités sportives), } \\
\text { - } \mathbf{4} \text { travailleurs indépendants (EURL, service sportif et hébergement), } \\
\text { - } \mathbf{3} \text { regroupements de travailleurs indépendants (EURL, SARL, monoactivité sportive), } \\
\text { - } \mathbf{4} \text { entreprises hôtelières (SARL, SA, service d'accueil), } \\
\text { - } \mathbf{4} \text { entreprises associatives et commerciales (Loi 1901, SMEL, services sportifs et d'accueil). }\end{array}$} \\
\hline
\end{tabular}

EURL : entreprise unipersonnelle à responsabilité limitée.

SARL : société anonyme à responsabilité limitée.

$\mathrm{SA}$ : société anonyme.

SEML : société d'économie mixte locale.

b) la compréhension des démarches de «conception-production» et de commercialisation. L'objectif était de comprendre le déroulement et le fonctionnement de l'action commerciale à travers l'analyse du marketing mix (élaboration des produits, fixation des prix, etc.).

Le traitement des données s'est fait, en premier lieu, thème par thème, dimension par dimension en se référant au repérage des indicateurs énoncés dans le cadre théorique ( $c f$. tableau 1). En second lieu, il s'agissait de croiser les différents thèmes et dimensions pour en sortir les éléments les plus structurants permettant la construction d'une typologie multicritères.

L'analyse des données empiriques, issues d'une recherche doctorale (Bouhaouala, 1999), relève quatre types de dirigeants d'entreprise qui correspondent à quatre types d'offre de services. Ces derniers se distinguent par des logiques d'action et des démarches commerciales spécifiques et relatives à leurs micromentalités. Ils se répartissent comme suit: 1) les indépendants passionnés (IP : 6/28), 2) les entrepreneurs indépendants (EI : 8/28), 3) les managers gestionnaires (MG:6/28) et 4) les conservateurs patrimoniaux (CP:7/28); enfin, un dirigeant présentant un profil hybride et soulevant des questions d'un intérêt théorique certain (passage d'un type à un autre) et qui fera l'objet d'une autre publication. 


\section{Micromentalités et actions commerciales des dirigeants de petites entreprises}

\section{L'offre artisanale du passionné}

\subsection{L'indépendant passionné (IP)}

Avant de se mettre à son propre compte, ce type de dirigeants est passé de la situation d'urbain salarié (hors secteur des services sportifs) à celui de travailleur saisonnier en passant par celui de pratiquant passionné. Il est donc passé de la phase du consommateur à celle d'offreur de services sportifs. Le transfert de compétences s'observe uniquement au niveau de la production d'un service sportif particulier (l'encadrement d'une spécialité sportive par saison, p. ex., le ski de fond hors piste).

La priorité pour ce dirigeant, c'est de vivre sa passion du sport et de la nature et de continuer à la cultiver. Ce qui se traduit par la recherche d'un travail alliant aspects ludique et convivial. En ce sens, la conception de l'activité professionnelle n'a rien d'un travail traditionnel contraignant (hiérarchie, horaires fixes, etc.). L'entreprise ne renvoie pas à une vision capitaliste (maximisation des profits financiers) ou entrepreneuriale (création et croissance). L'entreprise et le travail sont conçus comme de simples moyens d'existence. La première est conçue également comme un lieu de partage avec les clients d'une passion commune, ces derniers ayant souvent la même conception du sport et de la nature. Entre le client et le prestataire s'instaure alors un lien social qui place l'aspect commercial au second rang.

L'IP possède un rapport réaliste et distant avec l'argent qui représente alors une source possible d'aliénation. Faire de l'argent une finalité signifie devenir son esclave, ce qui semble contraire à ses valeurs fondamentales. Le «dilemme » que cela engendre est qu'il est à la fois conscient du rôle de l'argent dans la réalisation des objectifs personnels (non économiques) et des conséquences, sur son mode de vie, d'une soumission à la recherche de la maximisation des revenus (investir plus, travailler davantage, embaucher, etc.). Pour ces raisons, il le prend à son juste niveau pour garantir la liberté de ses actions. En d'autres termes, c'est une des conditions lui permettant d'assouvir sa passion et de subvenir à ses besoins.

\subsection{Démarche artisanale centrée sur la passion du sport}

La démarche de l'IP est basée sur une logique hédoniste singulière et une stratégie de production et de commercialisation centrée sur «soi » et sur sa passion. Fidèle au projet social visant à privilégier la qualité de vie sur les plateaux du Vercors en vivant de sa passion, ce type de dirigeants a mis en place une démarche commerciale non orientée par le marché ou le client. Elle se caractérise par une forte spécialisation dans une activité sportive correspondant à sa passion (soit l'encadrement

Revue internationale P.M.E., vol. 15, n $\mathrm{n}^{\circ} 1,2002$ 
du ski de fond hors piste). La spécialisation est également liée aux compétences élevées et à la très bonne connaissance du milieu naturel qui caractérisent ce dirigeant, qui est également un très bon pratiquant de sport de nature.

Elle permet de: $a$ ) personnifier le produit au sens de son identification au dirigeant passionné, $b$ ) de fidéliser une clientèle qui partage la même passion du sport et qui recherche ce type de savoir-faire et $c$ ) de retrouver un certain plaisir dans le travail (aspect ludique, relationnel, convivial, etc.). L'adéquation entre l'offre et la demande étant peut être ici expliquée par la recherche a priori de la satisfaction du client. Mais même si elle donne cette apparence, elle est le résultat d'une rencontre non recherchée entre prestataires et pratiquants passionnés (Lahlou et al., 1991) ou entre un savoir-faire unique et une demande spécifiques (Pacitto et Tordjman, 2000).

Dans la création et l'élaboration de ses services, le dirigeant passionné accorde une grande importance à sa propre conception du sport de nature, du tourisme de montagne et à son propre plaisir dans la pratique du sport. En effet, il imagine les services sportifs en fonction de ses goûts personnels, de sa manière de pratiquer et de sa propre expérience sportive. Ce rapport passionnel au service sportif se traduit par une apologie de la qualité et de l'authenticité de ses prestations. Il considère, d'un point de vue personnel, ses services comme étant originaux et de très bonne qualité. Le primat des critères personnels et passionnels dans l'élaboration des produits au détriment de critères commerciaux (demande, rentabilité, coûts, concurrence, etc.) renforce chez l'IP la personnification des produits commercialisés. Le fait du partage avec sa clientèle de la même passion (Lahlou et al., 1991) permet à ce type d'entreprise de rendre viable ce type de production. Le rapport passionnel au sport rapproche la démarche de l'offre passionnée de l'«idéologie artisanale » du travail bien fait (Zarca, 1986), bien que ce dirigeant soit plus centré sur sa passion de la pratique sportive que sur la création d'une « œuvre »; la dimension manuelle du travail est plus forte chez l'artisan que chez l'IP.

La fixation des prix n'est pas sans lien avec la forte personnification de la production qui constitue l'une des caractéristiques principales de ce type de dirigeants. En effet, la fixation des honoraires ou du prix à la journée ne se rapporte pas aux coûts de production (matériel, salaire, communication, etc.), mais plutôt à une estimation personnelle de la qualité et des besoins d'existence (tarif journalier minimum viable pour un dirigeant). Les arguments qu'il donne ne sont que des justifications a posteriori et non des indicateurs d'une démarche analytique et préalable des coûts. Les arguments liés à la qualité permettent de justifier les prix (s'ils apparaissent élevés) et le niveau de vie permet de justifier une limite minimale à observer.

L'IP ne se percevant pas dans un univers concurrentiel, la publicité est vécue comme un mal nécessaire et non pas comme un facteur de commercialisation essentiel au bon fonctionnement de l'entreprise. Dans un premier temps, on peut 
comprendre son rapport à la communication à partir de contraintes économiques liées à la très petite taille de cette dernière (EURL, CA $<$ à $0,5 \mathrm{MF}$, effectif $\leq$ à 1 salarié). Cependant la prise en compte de la micromentalité du dirigeant montre que les techniques du marketing ne correspondent ni à son goût, ni à ses objectifs (refus d'expansion et d'investissement, refus de travailler plus, etc.; Bouhaouala, 2001 ; Bouhaouala et Chifflet, 2001).

La démarche du passionné consiste alors à externaliser la publicité en se tournant vers des solutions collectives. Il s'agit de faire partie soit d'un réseau de professionnels extérieurs au Vercors (Gîtes de France, Gîtes Panda, Éducation et Nature, Association professionnelle du cheval, etc.), soit d'un réseau de professionnels locaux (bureau des guides, alliance de professionnels locaux, etc.) ou d'utiliser des supports classiques gratuits ou peu coûteux (organismes publics ou parapublics de promotion: Offices du tourisme, Comité départemental de tourisme, Association pour le développement du tourisme, etc.).

Cette externalisation permet au dirigeant de se centrer davantage sur sa passion (la pratique sportive) en s'appuyant sur des « réseaux » personnalisés lui permettant de préserver son indépendance. Sa relation avec le client est «fusionnelle » dans le sens où elle est vécue comme un partage et non comme un échange commercial. Ce qui traduit une adéquation a posteriori entre les attentes du client (goûts, manière de pratiquer, etc.) et celles du prestataire. La richesse «sociale» de la relation prestataire-client crée les conditions idéales à l'instauration de liens amicaux permettant une fidélité durable.

TABLEAU 2

Synthèse multicritère de l'IP

\begin{tabular}{ll}
\hline Micromentalité & Passionnelle \\
1) Le travail et l'entreprise & 1) Activité professionnelle ludique, lieu de partage de sa passion. \\
2) L'argent & 2) Moyens de subsistance et d'assouvissement de la passion. \\
3) Le sport et la montagne & $\begin{array}{l}\text { 3) Passion personnelle constituant la finalité } \\
\text { de l'activité professionnelle. }\end{array}$ \\
\begin{tabular}{ll} 
4) Le client & 4) Partageant la même conception du sport et de la montagne. \\
5) La concurrence & 5) Ignorée, car ne constituant pas \\
6) Objectif & 6) Vivre de sa passion, éviter la croissance de la microfirme. \\
\hline Action commerciale & Démarche artisanale centrée sur la passion \\
1) Production & 1) Orientée sur « soi » selon l'idéologie passionnelle. \\
2) Prix & 2) Estimation subjective non fondée sur un calcul analytique \\
3) Communication & des coûts. \\
4) Vente & 4) Fon nécessaire pour la réalisation des objectifs du dirigeant. \\
Logique d'action & Hédoniste
\end{tabular} \\
\hline
\end{tabular}

Revue internationale P.M.E., vol. 15, n $\mathrm{n}^{\circ} 1,2002$ 
Le dirigeant laisse apparaître cette adéquation comme «naturelle», car non recherchée dans un but commercial. En cela, l'offre artisanale du passionné se rapproche du marché du livre et de l'art telle que décrite par Lahlou et al. (1991).

\section{L'offre entrepreneuriale}

\subsection{L'entrepreneur indépendant (EI)}

Ce type de dirigeants est passé par le salariat et / ou par le statut de travailleur indépendant dans le secteur du plein air sur le Vercors. Il a occupé auparavant soit des fonctions de responsabilité ou d'organisation (directeur de station, responsable d'équipe, chargé de mission, etc.). Il connaît bien par conséquent le marché et ses rouages. On peut dire qu'il a bien préparé son opération et a bénéficié d'un transfert positif de compétences (gestion, organisation), de «connaissances » (réseaux) et d'expérience sportive. Son passage au statut d'entrepreneur s'est fait par la création avec des amis d'une agence de tourisme sportif. Cette forme «d'entrepreneuriat socialisé » est fondée sur un partage de la même vision du monde professionnel, mais elle permet également un partage des risques financiers et un élargissement du réseau des « connaissances utiles » (sous-traitants, partenaires, etc.).

Bien que recherchant la croissance, la rentabilité et le maintien de son affaire, ce dirigeant n'est pas conditionné par l'idéologie de «l'entrepreneur capitaliste » classique (Zarca, 1986). Ce dernier s'intéresse davantage à la valeur patrimoniale et très peu à la capacité de création de l'entreprise, alors que l'activité de l'EI est centrée sur le désir d'entreprendre et de faire pour lui-même. La rentabilité et l'argent ne représentent que des «instruments » d'action et la croissance de l'entreprise un «signe » de sa propre réussite sociale; son véritable objectif est lié à sa soif de se réaliser socialement. L'entreprise, perçue comme un lieu de création et d'action, répond à ce dernier objectif et lui permet de garantir son indépendance. Ce type d'objectif s'inscrit donc dans une vision individualiste en ce sens que la création de richesse, de rentabilité, d'emplois, etc., ne sont que des effets économiques engendrés et orientés par le projet personnel du dirigeant.

La conception du travail de l'entrepreneur indépendant est fondée sur des valeurs relatives à l'action et au fait d'entreprendre en toute indépendance (innovation, créativité, liberté, etc.). Ce qui explique son refus d'occuper un emploi de dirigeant salarié (même bien payé) et d'opter pour la configuration de l'entreprise par actionnariat. En effet, le salariat et l'actionnariat constituent à ses yeux des freins à sa liberté et à son épanouissement personnel.

L'entreprise possède deux fonctions : elle est une affaire qui doit rapporter de l'argent afin de bien en vivre et en même temps elle doit permettre son propre développement. Les revenus et la croissance que cette dernière produit sont 
donc des effets économiques attendus et recherchés par le dirigeant. En somme, le travail, l'entreprise et les revenus signifient respectivement « entreprendre, créer de la richesse et investir pour la croissance». Le travail n'est donc pas envisagé sous son aspect «ludique», ni l'argent sous l'aspect d'un "simple moyen de subsistance ». Le rapport à l'argent est plutôt « instrumentalisé », ce dernier jouant un rôle social (revenus personnels, signe de réussite, etc.), mais surtout économique (indicateur de rentabilité et instrument de croissance, etc.).

L'objectif de ce type de dirigeants étant d'entreprendre pour faire croître son affaire ${ }^{6}$ et ses parts de marché, la concurrence est sérieusement prise en compte. Parce qu'elle correspond à la compétitivité «stimulatrice» de l'action et compose l'idéologie entrepreneuriale à laquelle s'identifie le dirigeant, la concurrence est très largement surestimée par ce dernier (Bouhaouala, 2001).

\subsection{La démarche entrepreneuriale centrée sur le produit}

L'objectif de «réalisation de soi» constitue l'élément fondamental de la logique de l'entrepreneur indépendant. Le développement et la croissance de l'entreprise semblent n'être qu'une traduction de cette dernière qu'on qualifie ici de logique entrepreneuriale. L'analyse de l'action commerciale de ce type de dirigeants révèle une démarche plus orientée sur le processus de production (activités sportives, encadrement sportif, organisation d'événements, etc.) et, dans une moindre mesure, sur le client et le marché. En d'autres termes, la démarche commerciale n'est pas tirée par le marché ou centrée exclusivement sur le client. Elle est plutôt fondée sur la valorisation de la créativité, l'innovation, l'expérience (sportive et entrepreneuriale) et la maîtrise du processus de production des services sportifs. En ce sens, l'entrepreneur indépendant ne correspond pas à l'image du mercaticien (Marion, 1995), mais se rapproche plutôt de «l'entrepreneur technicien génial » (Bauer, 1993). Sa démarche d'élaboration des services sportifs est influencée par sa soif de créer, d'innover, d'entreprendre, etc., bref, par son désir d'accomplissement.

Ici la démarche commerciale s'oppose à celle du dirigeant passionné dans le sens où l'EI recherche l'innovation, qu'elle soit de produit ou de marché. Innover en matière de service (du point de vue sportif ou technologique), même en l'absence de gains de productivité ou de rentabilité élevée donne au dirigeant le sentiment d'entreprendre, donc d'exister. Ce type de dirigeants assoit sa démarche sur une approche empirique du marché et sur une interprétation subjective d'informations sur le tourisme d'une manière globale. Sa conviction est d'autant plus forte lorsque son interprétation coïncide avec l'idée qu'il se fait de l'avenir. L'absence de collecte

6. Lorsqu'il s'agit de croissance, l'organisation par actionnariat constitue la limite infranchissable par l'entrepreneur indépendant.

Revue internationale P.M.E., vol. 15, nº 1, 2002 
d'informations précises et formalisées concernant la demande ou le marché est un indicateur du recours au ressenti et à l'expérience, ainsi qu'à la «forte confiance en soi» (Julien et Marchesnay, 1996).

En raison de sa recherche de croissance, ce dirigeant est amené à prendre des risques en procédant à des investissements importants, ce qui engendre la nécessité de rentabilité. En conséquence, cette dernière joue un rôle non négligeable dans la démarche de fixation des prix. Les prix sont alors fondés sur : $a$ ) l'analyse, plus ou moins précise, des coûts de production, $b$ ) le calcul d'un seuil de rentabilité satisfaisante et $c$ ) la prise en compte du marché (concurrents et intermédiaires : les agences de voyages).

En ce qui concerne le calcul des coûts, le dirigeant intègre les paramètres économiques et comptables classiques : les investissements (locaux, matériel sportif, équipements divers, etc.) et les coûts généraux (salaires, entretien des équipements, matériel de bureau, véhicules, etc.). Cependant, bien que l'entrepreneur calcule relativement bien les coûts liés au produit, il éprouve de grandes difficultés à estimer et à intégrer le coût de son propre travail (temps passé à l'organisation, réunion, accueil, démarchage des clients, formation, etc.). En effet, il ne semble pas maîtriser tous les éléments de calcul liés à la fixation des prix. Le dirigeant passe beaucoup de temps au travail, s'investit à plusieurs niveaux (et dans de nombreuses tâches) et finit par ne plus savoir ce qu'il doit facturer aux clients.

Le rôle de la communication est indiscutable. Faire connaître les produits et l'entreprise est une opération indispensable. La preuve, les opérations de publicité et de communication constituent le troisième poste de dépenses après les salaires et les frais de fonctionnement. Les dépenses de communication sont estimées par le dirigeant entre $10 \%$ et $15 \%$ du chiffre d'affaires.

La démarche de communication est également fondée sur l'analyse empirique du secteur. Bien qu'il tente de faire une communication ciblée sur un segment de clientèle (l'entreprise spécialiste et concentrée) ou différenciée par segment de clientèle (l'entreprise diversifiée), le dirigeant ne fait référence à aucune étude de clientèle ou à des sources d'information fiables (formalisées et vérifiables) qui puissent justifier ses choix de communication (supports, arguments, techniques, etc.). Il s'appuie essentiellement sur le «flair» et l'expérience du terrain.

L'observation d'une dispersion non justifiée de supports de communication traduit cette soif de faire et d'entreprendre qui caractérise l'entrepreneur. La communication lui donne en quelque sorte l'impression de faire comme les grandes entreprises, ce qui est révélateur de la croyance de l'EI dans «l'idéologie de la grande entreprise». S'il utilise le langage «du parler seulement économique» (Bauer, 1993), cela ne veut pas dire qu'il applique systématiquement les principes de la rationalité économique. Mais c'est simplement pour se donner l'impression 
d'être un «vrai entrepreneur », conforme à l'image qu'il se fait de l'entreprise. Effectivement, cette logique d'imitation a même poussé ce type de dirigeants à recruter des commerciaux au profil du «mercaticien » provenant de grandes entreprises. Il joue en quelque sorte «à la grande entreprise» sans en avoir les capacités (EURL, SARL, CA compris entre 1 et 5 MF; effectif de 3 à 14 salariés) ni les caractéristiques.

Pour compenser ses lacunes en matière de commercialisation, l'entrepreneur indépendant met en œuvre des procédés particuliers. Travaillant le plus souvent avec des clientèles de groupe (entreprise, comité d'entreprise, séminaires, etc.), le « bouche à oreille » est insuffisant pour garantir le volume d'activité escompté. La commercialisation se fait alors en combinant quatre manières différentes : $a$ ) démarchage direct auprès des « gros clients », $b$ ) intermédiaires ou agences de voyages (donneurs d'ordres),c) réseaux spécialisés (sportifs, p. ex., organisations de vol libre) et d) réseaux locaux (p. ex., accord avec des entreprises hôtelières).

Cependant, l'effort de l'entrepreneur est plutôt porté sur la maîtrise de la variable «produit». Le savoir-faire et l'intérêt du dirigeant se situent davantage au niveau de la « conception-production » (innovation, création, organisation, etc.) et moins au niveau de la commercialisation. Bien que cette dernière soit le moyen central pour le développement économique de l'entreprise, l'orientation stratégique de l'EI est plus centrée sur le produit que sur le marché.

\section{TABLEAU 3}

\section{Synthèse multicritère de l'EI}

Micromentalité
1. Le travail et l'entreprise
2. L'argent
3. Le sport et la montagne
4. Le client
5. La concurrence
6. Objectif

\section{Action commerciale}

1. Production

2. Prix

3. Communication

4. Vente

Logique d'action
L'entrepreneur
1. Finalité, entreprendre, objet d'accomplissement et lieu de création.
2. Instrument économique et signe de réussite sociale.
3. Support de production et de créativité.
4. Consommateur.
5. Amplifiée, constitutive de l'idéologie de l'entrepreneur.
6. Réalisation de soi.

\section{Démarche entrepreneuriale centrée sur le produit}

1. Poussée par l'innovation technique (sportive) et technologique.

2. Estimation empirique fondée sur les coûts de production classiques.

3. Correspondant à l'objectif du dirigeant, externalisée en grande partie.

4. Partagée entre vente directe, intermédiaires (agences) et réseaux. 


\section{L’offre sociomanagériale}

\subsection{Le manager gestionnaire (MG)}

Le manager gestionnaire a accédé à la position de dirigeant (salarié) d'entreprise du secteur du tourisme de pleine nature par l'intermédiaire de la filière socioéducative et de réseaux de connaissances professionnelles. En effet, la première lui a permis d'acquérir un profil et une expérience de gestionnaire et les seconds lui ont rendu possible le recrutement sur le Vercors. Il a déjà exercé des fonctions de gestion et d'organisation, mais en dehors du secteur du tourisme sportif. Le transfert de compétences se situe au niveau du management et de l'organisation interne (gestion du budget, du personnel, marketing interne, etc.).

L'«esprit d'entreprise » de ce type de dirigeants ne correspond pas à celui du « capitaliste » (Zarca, 1986) ou de l'entrepreneur indépendant décrit plus haut. En effet, il se distingue de ce dernier, d'une part, par le fait qu'il est un dirigeant salarié par une petite entreprise du tourisme (entreprise associative, filiale d'un groupe [soit Maeva], SEML ${ }^{7}, \mathrm{CA}$ : 1,5 à $22 \mathrm{MF}$; effectif : 3 à 50 salariés) et, d'autre part, par son besoin «d'être socialement utile » à son environnement social (employeur, employés, vie locale, etc.). Sa capacité d'entreprendre, se limitant à des actions internes à l'organisation, le manager se met en quelque sorte à la disposition des autres pour la réalisation de projets socioéconomiques. De ce fait, on peut le qualifier «d'intrapreneur altruiste ». Le modèle de son entreprise ressemble à celui de l' « entreprise alternative » (Joyal, 1986; Julien et Marchesnay, 1988) et son mode de fonctionnement est fondé à la fois sur une «idéologie sociétale et managériale ». En effet, bien que les objectifs visés (économiques ou sociaux) dépendent du marché, ce dernier et l'entreprise sont perçus comme étant au service du social. En d'autres termes, même les objectifs économiques sont « retraduits » par le dirigeant en termes d'objectifs sociaux collectifs : emplois locaux, stimulation de la vie locale, démocratisation du tourisme, etc.

Contrairement au passionné et à l'entrepreneur, la conception du travail du manager se rapproche du monde du salariat traditionnel. Le manque d'autonomie, les contraintes d'horaires, de hiérarchies (conseil d'administration), l'effort, la responsabilité envers les autres, etc., sont acceptés et ne posent pas d'énormes problèmes. L'effort consenti dans le travail, bien que le dirigeant ne soit pas propriétaire de l'entreprise, s'explique par la volonté d'assumer ses responsabilités morales envers l'environnement social qui dépend directement ou indirectement de l'entreprise (l'employeur, le développement local, les emplois, la famille du dirigeant, etc.).

7. SEML: société d'économie mixte locale. Ici les principaux actionnaires privés sont composés de familles locales propriétaires d'entreprises et de commerces (hôtels, magasins d'articles de sport et de location, restaurants, etc.).

Revue internationale P.M.E., vol. 15, nº 1, 2002 
Le manager accorde donc une grande importance à la notion de «responsabilité » envers les autres. Il se présente non seulement comme responsable de l'entreprise (envers l'employeur), mais également des emplois et du tourisme pour tous (familles modestes, jeunes et enfants de milieux défavorisés, etc.). C'est ainsi que ce dirigeant justifie son engagement et son acceptation d'une autonomie relative dans son activité. Le travail semble prendre une dimension sociale importante.

La conception de l'argent est plus associée à «l'utilité sociale» qu'il représente. Son importance est liée à son usage social, son rôle économique est donc relégué au second rang. Les retombées socioéconomiques locales en termes d'emplois et de développement justifient la prise en compte de la rentabilité, de l'équilibre du budget, etc., dans son rapport à l'argent. Cependant, la richesse générée par l'entreprise n'est pas perçue prioritairement en termes de profits financiers ou d'accumulation du capital ; cette richesse est surtout associée aux résultats socioéconomiques et à son effet sur l'environnement social. Parce qu'il semble être persuadé du rôle moteur que joue l'entreprise dans la vie socioéconomique locale, le manager n'ignore pas l'importance économique de la rentabilité et son rôle dans le maintien de l'entreprise. Sans se détacher de la priorité du «bon usage » social de l'argent, il se montre convaincu que la rentabilité, le maintien et la croissance de l'entreprise représentent les signes d'une bonne gestion.

Par opposition aux deux types précédents, le manager ne se présente pas comme un passionné ou un spécialiste du sport de montagne, mais plutôt comme un dirigeant responsable d'un projet collectif dont le bon fonctionnement de l'entreprise fait partie. La montagne et le sport sont perçus comme des atouts commerciaux majeurs pour la réussite de l'entreprise. La montagne (le Vercors) constitue une force de vente, une valeur monnayable et les activités sportives sont « des produits d'appel ». Ces derniers sont des produits commerciaux spécifiques à la montagne, mais soumis à la loi universelle du marché. Le manager a le sentiment de les utiliser dans l'univers marchand, mais à des fins sociales et collectives.

Cela est une preuve de son faible attachement au Vercors en tant qu'espace de vie ou de pratique sportive. En conséquence, il est capable de se délocaliser si les conditions économiques ou si l'employeur l'imposaient. En effet, contrairement aux autres types de dirigeants, il n'est ni sportif, ni ancré dans le territoire local; son attachement est plus fort envers son travail, sa fonction sociale et son employeur.

\subsection{Démarche managériale centrée sur le marché}

Les stratégies mises en place par ce type de dirigeants sont fondées sur les objectifs sociaux (maintien des emplois, développement local, accès au tourisme) et économiques (rentabilité, équilibre financier, etc.). Le MG, ayant une conception plutôt commerciale du sport et du tourisme de nature, adopte une démarche commerciale orientée vers les clients et le marché. En d'autres termes, contrairement à l'EI qui

Revue internationale P.M.E., vol. 15, nº 1, 2002 
centre sa démarche sur l'élaboration des services, le manager fonde son action commerciale sur les paramètres du marché (coûts de production, faisabilité, concurrence, la demande, etc.). Il se réfère aux objectifs de l'employeur, aux besoins des clients et aux tendances du marché. Le dirigeant s'inscrit donc dans une logique managériale; la production doit se vendre et pour cela elle doit répondre aux critères de la concurrence et aux attentes des segments de clientèles.

Sa logique de production est déterminée par la prise en compte des coûts qui constituent l'élément central. En effet, lorsque le produit a rempli les conditions de faisabilité (techniques, législatives, sécuritaires, commerciales), il doit remplir la condition financière. En d'autres termes, si les coûts de production sont élevés par rapport au prix du marché et donnent des prix inadaptés au pouvoir d'achat de la clientèle visée, le produit sera abandonné.

L'adaptation aux différentes demandes des clients est fondée sur le recueil d'informations, lui-même s'appuyant sur des techniques simples mais rationalisées et objectivement fiables. L'information est prise directement au niveau de la clientèle, par communication directe avec le responsable lorsqu'il s'agit de groupes ou par questionnaires lorsqu'il s'agit de clients individuels. Ainsi, les critères de qualité ne sont pas estimés subjectivement du point de vue des dirigeants. Le manager adapte objectivement la qualité des produits selon le degré de satisfaction des clients et la pression concurrentielle.

Contrairement à l'entrepreneur, le manager attribue au prix un rôle économique plus important. Le prix est lié à la rentabilité financière, à l'équilibre budgétaire, aux parts de marché et aux segments de clientèles. Il est perçu comme un élément important dans le choix de la clientèle. Par conséquent, le prix représente un instrument stratégique central dans le comportement concurrentiel de ce type d'entreprises.

La communication est perçue comme un élément important dans la réalisation des objectifs de l'entreprise. En raison de la portée (nationale et régionale) du message recherché (commercial ou social), le manager fait appel à des « réseaux » spécialisés se distinguant par une grande capacité de vente et de communication. Ce moyen présente deux avantages. Premièrement, du point de vue managérial, les réseaux (professionnels, commerciaux, associatifs ou institutionnels) permettent : a) l'externalisation des opérations de communication, $b$ ) la baisse des coûts et c) le ciblage des clientèles recherchées (par le choix du réseau). Deuxièmement, sur le plan social, l'adhésion à tel ou tel réseau permet d'afficher les objectifs défendus (p. ex., le tourisme familial) et de bénéficier du soutien d'organisations portant les mêmes valeurs (p. ex., la Fédération des familles de France).

La stratégie commerciale est donc orientée par le projet socioéconomique de l'organisation. La démarche du dirigeant est fidèle à «l'idéologie sociétale et 
managériale » où tout est rationalisé et orienté sur deux objectifs apparemment contradictoires : social et économique. La réalisation des buts sociaux devient alors tributaire de l'efficacité économique et l'existence économique de l'organisation se justifie par les effets sociaux recherchés localement. Le marché est alors considéré comme un moyen indispensable pour la réalisation de la mission du dirigeant. Cette dernière n'est pas la moindre, car il est difficile de trouver « la logique alternative » au tout économique lorsque la pérennité de l'organisation dépend du marché. De ce fait, la logique sociomanagériale du manager tient compte des lois de la gestion managériale et des liens sociaux.

\section{TABLEAU 4}

\section{Synthèse multicritère du MG}

\begin{tabular}{|c|c|}
\hline Micromentalité & Managériale \\
\hline 1. Le travail et l'entreprise & $\begin{array}{l}\text { 1. Autonomie relative, responsabilité envers les autres, } \\
\text { outil de développement local. }\end{array}$ \\
\hline 2. L'argent & 2. Fonction sociale prioritaire. \\
\hline 3. Le sport et la montagne & 3. Produit d'appel et force de vente, des produits commerciaux. \\
\hline 4. Le client & 4. Touriste : « clients rois ». \\
\hline 5. La concurrence & 5. Une réalité constitutive de la stratégie commerciale globale. \\
\hline 6. Objectif & 6. Concilier logique sociale et économique : développement local. \\
\hline Action commerciale & Démarche managériale centrée sur le marché \\
\hline 1. Production & $\begin{array}{l}\text { 1. Tirée par le marché, la concurrence } \\
\text { et le segment de clientèle ciblé. }\end{array}$ \\
\hline 2. Prix & $\begin{array}{l}\text { 2. Selon les coûts de production, } \\
\text { la concurrence et le pouvoir d'achat des clients. }\end{array}$ \\
\hline 3. Communication & $\begin{array}{l}\text { 3. Externalisée, avec ciblage de clientèles } \\
\text { et à une échelle nationale. }\end{array}$ \\
\hline 4. Vente & $\begin{array}{l}\text { 4. Essentiellement par réseaux et intermédiaires } \\
\text { à force de vente élevée. }\end{array}$ \\
\hline Logique d'action & Sociomanagériale \\
\hline
\end{tabular}

\section{L'offre traditionaliste}

\subsection{Le conservateur patrimonial (CP)}

Le conservateur est originaire du Vercors. L'héritage de l'entreprise et du savoirfaire familial constitue le cœur de son patrimoine et représente la voie d'accès à la position de dirigeant. Le noyau dur de son activité est constitué par les métiers de l'accueil (hébergement, restauration) dont il a acquis les bases auprès des parents avec un court détour par les écoles hôtelières de la région. Les expériences professionnelles vécues se limitent donc à l'entreprise familiale. Le transfert de compétences s'est opéré par héritage et se situe au niveau de la production et de la gestion du patrimoine familial.

Revue internationale P.M.E., vol. 15, nº 1, 2002 
Le groupe des entreprises du conservateur est constitué en grande partie d'entreprises familiales hôtelières et de très peu de prestataires de services sportifs (SARL, SA, CA : 0,5 à $10 \mathrm{MF}$; effectif : 1 à 25 salariés). Les caractéristiques de son mode de gestion et de fonctionnement renvoient aux modèles développés par Bauer (1993), Gresle (1981) ainsi que Julien et Marchesnay (1988). En effet, son manque d'action entrepreneuriale le rapproche du modèle de «l'occupant de la maison familiale » (Bauer, 1993); sa défiance à l'égard du marché du travail et des capitaux constituent des points de similitude avec «l'entreprise de l'artisan » (Gresle, 1981) ; l'objectif de pérennisation de l'entreprise (dans le cercle familial) l'apparente au modèle de «l'entrepreneur PIC» (Julien et Marchesnay, 1988). Enfin, la recherche des profits et de l'accumulation du capital le rapproche du dirigeant de «l'entreprise artisanale capitaliste (Zarca, 1986).

La prédominance de la dimension patrimoniale chez le conservateur se traduit par des conceptions (de l'entreprise, du marché...) et des objectifs contradictoires par rapport aux principes de l'homo æeconomicus. Ces contradictions sont liées à la volonté morale de pérennisation de l'entreprise au sein du patrimoine familial et à la volonté économique de faire des profits financiers à court terme. En d'autres mots, le dirigeant de ce type d'entreprise est plus conservateur qu'entrepreneur.

La conception du travail est fortement liée à la dimension familiale et patrimoniale de l'entreprise. Le travail est perçu comme une «destinée », comme un sacrifice pour conserver le patrimoine familial. Ce type de dirigeants trouve son travail pénible, sans satisfaction personnelle ou directe, il n'est pas présenté comme un choix personnel. Le dirigeant conservateur y passe beaucoup de temps et accomplit un grand nombre de tâches (production, accueil, contacts avec l'environnement local, administrative, etc.) parfois répétitives, routinières, etc. Cela démontre également sa volonté de tout contrôler et d'indépendance à l'égard du marché du travail (limitation de l'embauche externe à la famille).

Le conservateur insiste sur l'aspect manuel et productif de son travail qui constitue le noyau dur de ses compétences. Le savoir-faire constitue le socle sur lequel se construit l'identité du métier. Le souci de maîtriser les principales fonctions le conduit à faire appel à l'emploi familial. Ce mode d'embauche devient alors un moyen de se décharger de certaines tâches (administratives, accueil) pour mieux se centrer sur la production de services.

La conception de l'argent est fortement liée à la valeur patrimoniale de l'entreprise qui a deux valeurs : financière et sociale. La première est estimée sur le marché des entreprises (terrains, bâtiments, équipements, clientèle, chiffre d'affaires, résultats, etc.) et la seconde est estimée par le dirigeant lui-même selon son histoire et ses projets de transmission, ainsi que selon le niveau de vie qu'elle procure à la famille. 
Quant à la montagne, sa conception est fondée sur trois dimensions différentes : patrimoniale, commerciale et en termes de cadre social de vie. Le Vercors étant le principal atout commercial, l'activité sportive est considérée comme une des déclinaisons possibles des potentialités de cet espace. Le sport est alors pensé comme un produit secondaire laissé aux entrepreneurs et autres passionnés. Cela correspond à une vision conservatrice conforme à la forme originelle du tourisme vécu sur le Vercors pendant l'époque faste comprise entre le début du siècle et les années 1980. Pour ce type de dirigeants, issu de vieilles familles locales (de montagnards), le Vercors est une partie constitutive du patrimoine des anciennes familles du massif, et ses retombées économiques doivent légitimement lui revenir.

\subsection{La démarche traditionaliste centrée sur le patrimoine}

L'analyse de l'action commerciale du conservateur révèle des contradictions entre l'objectif affiché et le comportement commercial concret. En effet, la démarche de production «traditionaliste» n'est pas orientée vers une recherche de croissance en termes de parts de marché ; elle est plus centrée sur la pérennisation du patrimoine familial (capitaux, savoir-faire, etc.) et le maintien des rentes qu'engendre leur affaire. La logique de production du conservateur ne peut pas être réduite à la recherche de bénéfices à court terme, cette dernière n'est qu'un effet de la première.

Ce type de dirigeants vise une rentabilité financière qui lui permet de maximiser ses revenus sous la contrainte du maintien de l'entreprise au sein du cercle familial. Les stratégies mises en œuvre sont alors fondées sur des ententes locales entre les anciennes entreprises familiales du Vercors. Ces ententes, qu'on pourrait qualifier de conventions, permettent au conservateur de réduire les risques financiers et d'éviter des schémas d'évolution pouvant le conduire vers les modèles de l'entreprise managériale ou de l'organisation entrepreneuriale. En effet, les conventions locales garantissent un revenu certain sous forme de rente et par là même atténuent la pression concurrentielle (Marchesnay, 1998). Contrairement à l'entrepreneur, chez le conservateur, cela se traduit par la recherche d'un taux de fréquentation (accueil ou services sportifs) satisfaisant sans procéder à des investissements lourds et incertains pouvant le conduire à une dépendance envers le marché des capitaux.

La démarche de production est fondée sur le savoir-faire hérité des parents et sur une vision traditionaliste du métier et des services commercialisés. Les éléments essentiels caractérisant sa démarche sont la qualité des services, de l'accueil, la satisfaction du «bon client» et la rentabilité financière à court terme.

L'estimation des critères de qualité se fait selon des normes d'appréciation classiques acceptées par l'ensemble du métier. En effet, le conservateur tient compte des critères définis par des organismes de labellisation (publics, des guides du voyageur, etc.) ou par des donneurs d'ordres (p. ex., agences de voyages). Aux yeux

Revue internationale P.M.E., vol. 15, nº 1, 2002 
du dirigeant, les normes de classement (hôtelière) ou de labellisation reconnues constituent le reflet idéal et indiscutable de la qualité de son travail, ainsi qu'un argument fort de sa tarification.

L'innovation et l'évolution du tourisme sportif de nature ne sont pas perçues comme une opportunité de développement, mais comme une menace pour son identité professionnelle (traditionnelle), donc pour son patrimoine. La croissance est associée à l'obligation d'embaucher des personnes étrangères au cercle familial, ce qui est perçu comme un «pas » vers la dépendance envers le marché du travail. Cela est révélateur d'une certaine aversion pour le changement.

Dans la démarche de fixation des prix, on constate trois éléments importants interreliés dans la logique du dirigeant conservateur. Le prix est considéré comme : 1) l'élément décisif du choix du client, 2) l'indicateur du niveau de qualité et 3) le garant de la rente familiale. De ce fait, la fixation des prix se déroule en trois étapes qui consistent : $a$ ) à intégrer ce que le $\mathrm{CP}$ appelle «le prix de revient », c'est-à-dire les charges fixes (salaires, fonctionnement, etc.) et les charges variables (achat de consommables : marchandises, etc.), $b$ ) estimer sa marge en fonction de la qualité et du client, ensuite $c$ ) se référer au point de vue des intermédiaires et aux conventions locales. Ce n'est que dans le cadre de ces dernières qu'il optera pour le meilleur prix lui permettant d'atteindre les revenus escomptés.

Le conservateur réalise très peu de publicité par ses propres moyens. Son action dans ce domaine se limite à une opération d'information auprès du client une fois le contact établi (répondre au téléphone, envoi de dépliants, de photographies, etc.). Intégrer les opérations de publicité dans les fonctions de l'entreprise est perçu comme une démarche non conforme à l'identité de son métier. Il s'appuie alors sur 1) la notoriété de son savoir-faire, 2) la capacité d'attraction du Vercors et 3) la force de vente des intermédiaires situés hors Vercors.

La vente et la commercialisation des produits dépendent donc en grande partie de réseaux formels (central de réservation, agences de voyages, intermédiaires) et de réseaux informels locaux (ententes locales). Ces dernières consistent en l'établissement d'accords tacites de partenariats entre les entreprises hôtelières et les entreprises sportives (Bouhaouala, 2001). Les termes du partenariat reposent sur un échange de clientèle dans le respect de certaines règles d'accueil et / ou de prix préférentiels.

En raison de son évitement des risques financiers, ce type de dirigeants préfère rester dans un schéma qu'il connaît bien et qui correspond le mieux à ses compétences. La solution qui lui semble donc la plus adéquate est l'externalisation de la communication et de la commercialisation vers les réseaux spécialisés. La stratégie du conservateur ne semble pas résulter d'une approche managériale, mais du souci de garantir une bonne rente en adoptant une démarche qui ne remet pas en question 
son objectif principal : le maintien de l'entreprise au sein du cercle familial. Son action commerciale est donc fondée sur la logique patrimoniale conduisant à une contradiction entre l'objectif commercial à court terme (bénéfices) et l'évitement du changement traduisant la prévention des risques de dépendance ou de perte du patrimoine familial. L'objectif moral à long terme (transmettre le patrimoine familial) détermine donc l'objectif économique à court terme.

\section{TABleau 5}

\section{Synthèse multicritère du CP}

\begin{tabular}{|c|c|}
\hline Micromentalité & Conservatrice \\
\hline 1. Le travail et l'entreprise & 1. Savoir-faire familial, patrimoine familial, source de revenus. \\
\hline 2. L'argent & 2. Bénéfices à court terme, rente familiale. \\
\hline 3. Le sport et la montagne & 3. Atout commercial, le Vercors fait partie du patrimoine familial. \\
\hline 4. Le client & 4. Clients : touriste séjournant et / ou dépensant localement. \\
\hline 5. La concurrence & $\begin{array}{l}\text { 5. Atténuée par des conventions et ententes locales } \\
\text { entre conservateurs. }\end{array}$ \\
\hline 6. Objectif & 6. Conserver l'entreprise au sein de la famille. \\
\hline Action commerciale & Démarche traditionaliste centrée sur le patrimoine \\
\hline 1. Production & $\begin{array}{l}\text { 1. Selon le savoir-faire traditionnel, les intermédiaires, } \\
\text { la demande. }\end{array}$ \\
\hline 2. Prix & $\begin{array}{l}\text { 2. Selon les coûts de production, le client } \\
\text { et les conventions locales. }\end{array}$ \\
\hline 3. Communication & 3. Externalisée, ne fait pas partie du métier. \\
\hline 4. Vente & 4. Essentiellement par réseaux et intermédiaires. \\
\hline Logique d'action & Patrimoniale \\
\hline
\end{tabular}

\section{Conclusion}

Cette analyse empirique a permis de montrer, d'une part, que les actions commerciales des dirigeants de petites entreprises du tourisme sportif ne correspondent pas exclusivement au modèle du mercaticien et, d'autre part, que le consommateur n'est pas systématiquement au centre de leurs stratégies commerciales. Au contraire, les résultats auxquels aboutit cet article révèlent une diversité de stratégies et de démarches commerciales correspondant à une diversité de conceptions, d'objectifs et de logiques d'action spécifiques à des groupes de dirigeants.

Cette variété des logiques de fonctionnement des dirigeants permet donc de poser la question de l'apport de telles approches aux analyses de l'activité commerciale des entreprises en question. En effet, sans remettre en question les principes théoriques du marketing management, l'objectif est de montrer que la prise en compte de variables et de modèles sociologiques dans une perspective non normative permet de comprendre autrement les actions commerciales des petites entreprises et d'expliquer leur pluralité. En ce sens, la notion de «mix marketing » a été

Revue internationale P.M.E., vol. 15, nº 1, 2002 
utilisée ici comme une grille de départ pour décrire les comportements relatifs à la production-commercialisation des services sportifs sans que les variables commerciales en soient les éléments exclusifs dans l'explication. Également, le modèle du mercaticien n'a pas été considéré comme le canon auquel les entrepreneurs doivent obligatoirement correspondre pour qu'ils soient considérés comme " performants ».

Ainsi, au regard de la typologie identifiée, on peut dire qu'il n'y a pas, d'un côté, un type d'acteurs économiques rationnels (le MG) et, de l'autre, des types de dirigeants plus ou moins « irrationnels » (l'IP, l'EI et le CP). Le comportement du manager qui apparaît, à un premier niveau de lecture, comme le plus proche de la rationalisation économique, est à réinterpréter à partir de sa culture managériale, mais également des objectifs sociaux qui le définissent. Dit autrement, chaque type de dirigeants possède sa propre rationalité qui ne renvoie pas exclusivement à celle de l' homo æeconomicus ou à celle du mercaticien, même si la part mercantile dans leur comportement n'est pas négligeable.

Il n'est donc pas possible d'évaluer les performances de ces entreprises prioritairement et exclusivement à partir des critères économiques (rentabilité, dynamisme de l'investissement, etc.) ou marketing classiques (satisfaction du client, concurrence, etc.). Il est clair, bien que constituant des éléments de leurs actions que, ni le passionné, ni le conservateur, ni même le manager et, dans une certaine mesure, l'entrepreneur ne conçoivent la maximisation des profits et la satisfaction du client comme des objectifs majeurs. Ces paramètres économiques sont souvent perçus comme des moyens pour atteindre un but idéal ${ }^{8}$ (Boutillier et Uzunidis, 1995). Même lorsque le langage économique et marketing est explicitement utilisé, celui-ci consiste davantage en une rhétorique exprimant l'idéologie et l'identité du dirigeant (soit l'entrepreneur ou le manager) ainsi que son appartenance à l'univers du marché. Ce dernier accède alors au statut de véritable croyance à partir de laquelle le dirigeant oriente ses actions et finit par se conformer à l'idée qu'il se fait des vertus de la concurrence par exemple, même si cette dernière est « objectivement » faible (Bouhaouala, 2001).

En somme, l'apport de l'analyse sociologique s'appuyant sur les micromentalités des acteurs économiques peut s'observer à deux niveaux : 1) la compréhension de la diversité des pratiques commerciales et 2) l'adaptation des formations

8. Les paramètres économiques (rentabilité, équilibre financier, etc.) ou marketing (satisfaction du client, évitement de la concurrence, part de marché, etc.) représentent pour les petites entreprises et leurs dirigeants des moyens ou des conditions de réaliser leur propre projet socioprofessionnel. En ce sens, sans négliger la part marchande dans leur comportement, leur finalité n'est pas exclusivement mercantile et contrairement à la grande entreprise par actionnariat, la configuration de leur entreprise est elle-même liée à leur mentalité et logique d'action (Bouhaouala, 2001; Bouhaouala et Chifflet 2001; Boutillier et Uzunidis, 1995 ; Raveyre et Saglio, 1984). 
et des programmes d'aide aux dirigeants de PE-TPE du tourisme sportif. L'évaluation de l'efficience de ces dernières devrait notamment s'appuyer sur des critères différenciés introduisant des variables non économiques. Ainsi, l'apparente «irrationalité » des comportements de certains types de dirigeants renverrait moins à un manque de compétences managériales et davantage à un ajustement entre logique d'action et pratiques commerciales spécifiques. On peut donc supposer qu'une offre de formation aux techniques commerciales adaptée aux projets et mentalités des dirigeants serait plus efficace. Il resterait, à partir de cette typologie, à poser les questions, d'une part, de l'apport des approches sociologiques aux analyses des activités de la petite entreprise et, d'autre part, de l'utilité de programmes d'aide et de formation différenciés.

En conclusion, un programme de recherche pluridisciplinaire, impliquant la sociologie et le marketing peut présenter un intérêt heuristique certain qu'il convient d'exploiter. Il suppose l'identification de critères (socioéconomiques) de réussite et / ou d'échec spécifiques aux actions commerciales des PE-TPE et leur croisement avec le contenu des modèles marketing plus conçus à partir de la grande entreprise et pour elle. Cette confrontation permettra de tester l'adaptation des modèles de gestion modernes et de mettre en perspective des techniques marketing ou des plans d'actions commerciales adaptés aux PE-TPE.

\section{Bibliographie}

BAUER, M. (1993), Les patrons de PME entre le pouvoir, l' entreprise et la famille, Paris, InterÉditions.

Bentabet, E., S. Michun et P. TrouvÉ (1999), Gestion des hommes et formation dans les très petites entreprise, Centre d'études et de recherches sur les qualifications, Étude $\mathrm{n}^{0} 72$.

Berett, T., T.L. BuRTON et T. SLACK (1993), «Quality products and quality services : factors leading to entrepreneurial success, sport and leisure industry », Leisure Studies, no 12, p. 93-106.

BERTRAND, N. (1996), L'ancrage spatial des entreprises en milieu rural : de l' espace fonctionnel à l'espace territoire, Thèse de doctorat, Université Pierre-Mendès-France, Grenoble II.

Bouhaouala, M. (1999), Micro-mentalités et logiques d' action des dirigeants des petites entreprises du tourisme sportif, Thèse de doctorat, Université Joseph-Fourier, Grenoble I.

Bouhaouala, M. (2001), «Relations inter-entreprises dans un marché local: le cas des PE-TPE du tourisme sportif en Vercors », Revue Espaces et Sociétés (sous presse).

Bouhaouala, M., et P. Chifflet (2001), «Logiques d'action des moniteurs des sports de pleine nature : entre passion et profession », Revue STAPS, n ${ }^{\circ} 57$.

Boutillier, S. et D. UzUnidis (1995), L'Entrepreneur, une analyse socioéconomique, Paris, Economica.

Revue internationale P.M.E., vol. 15, nº 1, 2002 
Boutillier, S. et D. Uzunidis (1999), La légende de l' entrepreneur, Paris, La Découverte et Syros.

Callot, P. (1999), «Un marché trop émietté pour être international», Espaces nº 157, p. 37-41.

CANDAu, P. (1981), « Pour une taxonomie de l'hypofirme », Revue d'économie industrielle, $\mathrm{n}^{\mathrm{o}} 16, \mathrm{p} .17-33$

Chantelat, P. (1995), «Stratégies mondiales des industries du sport et homogénéisation de la consommation sportive : Sport, relations sociales et action collective », Bordeaux, Maison des sciences de l'homme d'Aquitaine, p. 647-656.

Cliquet, G. (1999), «Marketing», dans R. Le Duff (dir.), Encyclopédie de la gestion et du management (E.G.M.), Paris, Dalloz.

Collins, M. et L. RANDOLPH (1991), « Business or hobby? Small firms in sport and recreation », dans A.J. Veal et P. Jonson (dir.), Leisure and Tourism : Social and Economic Change, Sidney, University of Technology, p. 433-438.

D'IRIBARNE, P. (1995), «La science économique et la barrière du sens », dans A. Jacob et H. Verin (dir.), L'inscription sociale du marché, Paris, L'Harmattan.

EIGLIER, P. et E. LANGEARD (1991), Servuction, le marketing des services, Paris, McGrawHill.

FLIPO, J.-P. (1999), « Activités de service et relations inter-entreprises : vers une gestion stratégique des facteurs relationnels et des éléments d'interface », Revue française de marketing, $\mathrm{n}^{\circ} 171$, p. 63-76.

FliPO, J.-P., X. AuZOuY, G. BENOIST et M. BICHERON (1980), Pratique de direction commerciale marketing, Paris, Les Éditions d'Organisation.

Freund, J. (1966), Sociologie de Max Weber, Paris, Presses universitaires de France.

GIARD, D. (1997), «Les enjeux du tourisme sportif de nature en montagne », Les Cahiers d'Espaces, no 52, p. 48-57.

Gislain, J.J. et P. STEINER (1995), La sociologie économique 1890-1920, Paris, Presses universitaires de France.

Granovetter, M. (1995), «La Notion d'embeddedness », dans A. Jacob et H. Vérin (dir.), L'inscription sociale du marché, Paris, L'Harmattan.

GRESLE, F. (1981), «L'indépendance professionnelle, actualité et portée de concept dans le cas français », Revue française de sociologie, XXII-4, p. 483-501.

Herscovici, A. (1994), Économie de la culture et de la communication, Paris, L'Harmattan.

Hirschman, A.O. (1995), Défection et prise de parole, Paris, Fayard.

Huberman, A.M. et M.B. Mills (1991), Analyse des données qualitatives, recueil de nouvelles méthodes, Belgique, De Boeck Université.

JoYAL, A. (1986), «Les difficultés de travailler autrement au Québec », Loisir et Société, vol. $9, \mathrm{n}^{\mathrm{o}} 2$.

Julien, P.-A. (1997), Les PME : bilan et perspectives, Québec, Les Presses Inter Universitaires.

JULIEN, P.-A. et M. MARChESNAY (1988), La petite entreprise : principes d'économie et de gestion, Paris, Vermette/Vuibert.

Revue internationale P.M.E., vol. 15, nº 1, 2002 
Julien, P.-A. et M. MARChESNAY (1996), L'Entrepreneuriat, Paris, Economica. Kotler, P. et B. Dubois (1989), Marketing management, Paris, Éditions Publi-Union.

LAHIRE, B. (1998), L'homme pluriel : les ressources de l'action, Paris, Nathan.

LAHLOU, S., J. MAFFrE et P. MOATI (1991), Régulation des marchés culturels : le rôle de la passion, Paris, CREDOC.

Lloyd, G.-E.-R. (1996), Pour en finir avec les mentalités, Paris, La Découverte.

LOVELOCK, C. et D. LAPERT (1999), Marketing des services : Stratégie, outils, management, Paris, Éditions Publi-Union.

MARCheSnAy, M. (1997), «La moyenne entreprise existe-t-elle? » Revue française de gestion, $\mathrm{n}^{\circ} 116$, p. 85-94.

MARChesnay, M. (1998), «Confiances et logiques entrepreneuriales », Économies et Sociétés, Série S.G., n ${ }^{\text {os }} 8-9$.

MARION, G. (1990), Marketing : mode d'emploi, réédition 1998, Paris, Éditions d'Organisation.

MARION, G. (1995), « Le marketing management en question », Revue française de gestion, $\mathrm{n}^{\mathrm{o}} 102$, p. $15-30$.

MARION, G. (1999), «La nouvelle crise des modèles rationalisateurs du marketing », Revue française de gestion, $\mathrm{n}^{\circ} 125$, p. 81-89.

MoAti, P. (1993), «Rationalité et marché des biens culturels », Problèmes économiques, $\mathrm{n}^{\circ} 2339$, p. 23-32.

Mucchielli, A. (1985), Les mentalités, Paris, Presses universitaires de France.

PACITTO, J.-C. et F. TORDJMAN (2000), « Très petite entreprise et marketing: les causes d'un malentendu », Revue internationale PME, vol. 13, n ${ }^{\text {os }} 3-4$, p. 37-62.

Pigeassou, C. (1997), «Sport et tourisme : Émergence du sport dans l'offre touristique, entre passion et raison », Cahier Espaces, n 52, p. 15-24.

Pigeassou, C. et R. Fergusson (1997), « Du concept de stratégie au management stratégique des organisations de services sportifs », dans C. Pigeassou et C. Garrabos (dir.), Management des organisations de services sportifs, Paris, Presses universitaires de France, p. 128-154.

PORTER, M. (1980), Choix stratégiques et concurrence, réédition 1990, Paris, Economica.

RAVEYRE, M.-F. et J. SAGLIO (1984), «Les systèmes industriels localisés : éléments pour une analyse sociologique des ensembles de PME industrielles », Sociologie du travail $\mathrm{n}^{\mathrm{o}} 2$, p. 157-176.

RuIz, G. (1997), «Les évolutions des pratiques sportives depuis dix ans, les grandes tendances actuelles », Cahier Espaces n ${ }^{\circ}$ 52, p. 8-14.

SAn José, A., P. Fidelle et G. BRuANT (1995), «Les paradoxes de l'enseignement du parapente », Revue STAPS, no 37, p. 7-18.

STEINER, P. (1998), Sociologie de la connaissance économique : essai sur les rationalisations de la connaissance économique (1750-1850), Paris, Presses universitaires de France.

SWEDBERG, R. (1990), «Vers une nouvelle sociologie économique », Revue du Mauss, no 9 , p. 33-70.

SWEDBERG, R. (1994), Une histoire de la sociologie économique, Paris, Desclée de Brouwer.

Revue internationale P.M.E., vol. 15, $\mathrm{n}^{\circ}$ 1, 2002 
WEBER, M. (1905), L'éthique protestante et l' esprit du capitalisme, réédition 1964, Paris, Plon. Weber, M. (1922), Économie et société, réédition 1995, Paris, Plon.

ZARCA, B. (1986), L'artisanat français, du métier traditionnel au groupe social, Paris, Economica.

ZELIZER, V. (1992), « Repenser le marché, la construction sociale du marché des bébés aux États-Unis, 1870-1930 », Actes de le recherche en sciences sociales, $n^{\circ}$ 94, septembre. 